\title{
COMPLEX SEQUENCING PROBLEMS
}

\section{Johann Hurink, Universität Osnabrück}

A complex sequencing problem may be formulated as follows. Let $X$ be a subset of all permutations of a finite set. Associated with each permutation $\pi$ in $X$ there is an optimization problem $O P T(\pi)$. Let $f(\pi)$ be the optimal solution value of $O P T(\pi)$. Find a permutation $\pi^{*}$ which optimizes $f$ over all permutations in $X$. Usually $O P T(\pi)$ is a problem which can be solved efficiently (e.g. a linear program, a shortest path problem etc.)

We will present some practical problems which may be formulated as complex sequencing problems. Among them there are machine scheduling problems, railway scheduling problems, the problem of finding placement sequences and bin locations for robots, tool switching problems on flexible machines, etc. Most of these problems arise in connection with flexible manufacturing.

In general complex sequencing problems are NP-hard although each optimization problem $P(\pi)$ is efficiently solvable. Therefore we used local search techniques to calculate heuristic solutions for complex sequencing problems. These techniques yields very good results for a wide class of problems. The best known local search techniques are iterative improvement, simulated annealing and tabu search. The most important step when designing a local search method is the definition of a neighborhood. A neighborhood is given be a set of moves which allows to calculate for each permutation $\pi$ a set $N(\pi)$ of neighbors. The iterative improvement method replaces the current permutation by its best neighbor and iterates this process until there is no neighbor which improves the current solution. The disadvantage of this method is the possibility to be trapped in a local minimum which is arbitrarily bad. Simulated annealing avoids this by with certain probability allowing moves which do not improve the current solution . Unfortunately simulated annealing is a very slow method. A better choice is the tabu search method.

We report on experience with local search applied to complex sequencing problems. 